\title{
Nasal carriage of methicillin-resistant Staphylococcus aureus in university students
}

Authors

Karina Aparecida Prates ${ }^{1}$

Ana Maria Torres ${ }^{1}$

Lourdes Botelho Garcia ${ }^{1}$

Sueli Fumie Yamada

Ogatta $^{2}$

Celso Luiz Cardoso ${ }^{1}$

Maria Cristina Bronharo

Tognim $^{1}$

${ }^{1}$ Department of Clinical Analyses, Universidade

Estadual de Maringá, PR,

Brazil.

${ }^{2}$ Department of

Microbiology, Universidade

Estadual de Londrina,

Londrina, PR, Brazil.
Submitted on: 06/30/2009 Approved on: 09/9/2009

\section{Correspondence to:}

Dra. Maria Cristina

Bronharo Tognim

Laboratório de

Microbiologia,

Departamento de Análises

Clínicas, Universidade

Estadual de Maringá,

Maringá - PR - Brazil

CEP: 87010-900

Phone: +55-44-32614952

Fax: +55-44-32614860

E-mail:

mcbtognim@uem.br

\section{This work was} supported in part by the Coordenação de Aperfeiçoamento do Pessoal de Ensino Superior (CAPES-Brazil).

\begin{abstract}
In a study of university students, the percentage nasal carriage of Staphylococcus aureus was $40.8 \%$ $(102 / 250)$. Of the isolates, $\mathrm{MIC}_{50}$ of methicillin was $0.5 \mu \mathrm{g} / \mathrm{mL}$ and $\mathrm{MIC}_{90}$ was $1 \mu \mathrm{g} / \mathrm{mL}$. Six (5.8\%) isolates were methicillin-resistant and carried the mecA gene. These results suggest that communityassociated methicillin-resistant $S$. aureus may be spreading in Brazil.
\end{abstract}

Keywords: nasal carriage, CA-MRSA, S. aureus.

[Braz J Infect Dis 2010;14(3):316-318]@Elsevier Editora Ltda.

Methicillin-resistant Staphylococcus aureus (MRSA) is an important cause of health careassociated infections worldwide. ${ }^{1}$ In recent years, cases of MRSA infection have been reported more frequently in healthy community individuals with no traditional risk factors for MRSA infection. ${ }^{2}$ These infections, apparently acquired in the community, are termed community-associated MRSA infections. ${ }^{2,3}$

Community-associated MRSA (CA-MRSA) strains differ from health care-associated (HA) MRSA strains in terms of epidemiology, microbiology, and clinical manifestations. ${ }^{3}$ CA-MRSA strains are generally susceptible to most antibiotics, contain staphylococcal chromosome cassette mecA type IV, produce the virulence factor Panton-Valentine leukocidin, and cause mainly skin and soft-tissue infections. ${ }^{2,4}$

It is well recognized that nasal carriage of MRSA represents a major risk factor for subsequent infection and transmission of this pathogen. ${ }^{5,6}$ Although several studies have reported the prevalence of MRSA nasal carriage in patients in health care-settings, ${ }^{7,8}$ this subject has been little investigated in healthy individuals in the broader community, ${ }^{9}$ and is practically unstudied in Brazil.

In this study, we determined the prevalence of nasal carriage of $S$. aureus in university students. Antibiotic susceptibility patterns, minimal inhibitory concentration to methicillin, and mecA gene detection of the isolates were included.
Volunteers - The volunteers were 250 healthy adults, 93 males and 157 females, including first- and second-year undergraduate students in pharmacy, nursing, dentistry and medicine at a Brazilian university. Their ages ranged from 18 to 27 years. After giving their written informed consent, the volunteers completed a questionnaire to obtain demographic data and information on risk factors for MRSA infection. The study was approved by the Institutional Review Board of the State University of Maringá.

Specimen collection - Each volunteer had one nasal specimen collected from the anterior nares, with a dry swab (Copan Diagnostics, Corona, CA, USA). Swabs were transported in Amies medium and processed within $2 \mathrm{~h}$ of collection. All swabs were collected by the same investigator during the study, which was conducted from February to June 2007.

Culture methods - The swab was first inoculated directly on mannitol-salt agar (MSA) (Difco Laboratories, Sparks, MD, USA). It was then placed in a tube containing $10 \mathrm{~mL}$ of tryptic soy broth (TSB) (Difco) supplemented with $7.5 \%$ sodium chloride (enrichment broth), which was subcultured on MSA after $24 \mathrm{~h}$. Inoculated plates were screened at 24,48 , and 72 h for typical colonies of staphylococci. S. aureus was identified by colonial morphology, Gram stain, and tube coagulase test.

Antimicrobialsusceptibilitytesting-The antimicrobial susceptibility of $S$. aureus isolates was 
assessed by disk-diffusion tests, according to the Clinical Laboratory Standards Institute guidelines. ${ }^{10,11}$ The following antimicrobialdiskswere used:penicillin (10U), oxacillin $(1 \mu \mathrm{g})$, ciprofloxacin $(5 \mu \mathrm{g})$, gentamicin $(10 \mu \mathrm{g})$, amikacin $(30 \mu \mathrm{g})$, telithromycin $(15 \mu \mathrm{g})$, linezolid $(30 \mu \mathrm{g})$, trimethoprim-sulfamethoxazole (1.25-23.75 $\mu \mathrm{g})$, and vancomycin $(30 \mu \mathrm{g})$. All the disks were obtained from Oxoid (Oxoid, Basingstoke, UK). A mupirocin $(5 \mu \mathrm{g})$ disk (Oxoid) was also tested, according to Finlay et al. (1997). ${ }^{12}$

Minimal inhibitory concentration (MIC) - MIC to oxacillin was assessed by both E-test ${ }^{\circledR}$ oxacillin strips (AB-Biodisk, Solna, Sweden) according to the manufacturer's instructions, and the agar dilution method as recommended by the Clinical Laboratory Standards Institute. ${ }^{11,13}$

Detection of mecA by polymerase chain reaction (PCR) - One hundred and two strains of $S$. aureus were analyzed by PCR assay, using total DNA after boiling the bacterial cells. The mecA gene was detected using $m e c A$ sense 5'TGGCTATCGTGTCACAATCG3' and mecA antisense 5'CTGGAACTTGTTGAGCAGAG3' primers. Cycling parameters were $94^{\circ} \mathrm{C}$ for $5 \mathrm{~min}$ followed by 30 cycles of $94^{\circ} \mathrm{C}$ for $30 \mathrm{sec}$, annealing at $52^{\circ} \mathrm{C}$ for $30 \mathrm{sec}$, extension at $72^{\circ} \mathrm{C}$ for $30 \mathrm{sec}$, and a final $7 \mathrm{~min}$ incubation at $72^{\circ}$ C. The amplification products ( $309 \mathrm{bp}$ ) were analyzed by electrophoresis in $1 \%$ agarose gel stained with ethidium bromide.

S. aureus was isolated from nasal swabs of $102(40.8 \%)$ of the 250 volunteers, and six (2.4\%) of them were CAMRSA carriers. None of the volunteers had any identified risk factor.

Recovery of $S$. aureus strains from volunteers' nostril by direct plating on MSA was $26.8 \%$ (67 of 250 ), and by enrichment broth was $40.8 \%$ (102 of 250 ). All the volunteers detected as nasal carriers of $S$. aureus by the MSA method, were also detected by the enrichment broth method.

All the 102 strains of $S$. aureus were sensitive to vancomycin, telithromycin, linezolid, gentamicin, and trimethoprim-sulfamethoxazole. Resistance to penicillin G, ciprofloxacin, oxacillin, and amikacin was found in $92.0 \%, 8.8 \%, 5.8 \%$, and $4.0 \%$ of the strains, respectively. Mupirocin resistance was detected in 6 of $102(5.8 \%)$ isolates, which were sensitive to oxacillin.

Of the 102 strains, the minimal inhibitory concentration to oxacillin ranged from $0.06 \mu \mathrm{g} / \mathrm{mL}$ to $256 \mu \mathrm{g} / \mathrm{mL}$, and $\mathrm{MIC}_{50}$ and $\mathrm{MIC}_{90}$ were $0.5 \mu \mathrm{g} / \mathrm{mL}$ and $1.0 \mu \mathrm{g} / \mathrm{mL}$, respectively. Six strains (5.88\%) showed MIC $\mu 32 \mu \mathrm{g} / \mathrm{mL}$ and carried the mecA gene, and were therefore considered CA-MRSA. Two CA-MRSA strains were recovered only by the enrichment broth method. E-test ${ }^{\circledR}$ and agar dilution methods gave similar results.
The prevalence of $S$. aureus nasal carriage varies according to the quality of sampling, culture techniques, and the population studied. ${ }^{5,9}$ Early cross-sectional surveys on nasal carriage demonstrated a mean carriage rate of $37.2 \% .{ }^{5}$ More recent studies have reported rates of approximately $27 \%$ in healthy adult populations. ${ }^{6,14}$ Two studies with pre-clinical medical students showed that $35.2 \%$ and $29 \%$ were $S$. aureus nasal carriers. ${ }^{15,16}$ Our results are consistent with these findings.

Although CA-MRSA emerged as a cause of infection in the community in the 1990s, the first report of infections caused by this microorganism in Brazil was published in $2005 .{ }^{17}$ Our study found the prevalence of CAMRSA nasal carriage in our student community to be $2.4 \%$, which is higher than the findings of similar studies by investigators outside Brazil. ${ }^{9,16,18}$

In cross-sectional studies, the choice of the microbiological method used for $S$. aureus carriage detection is important, because the nasal culture is done only once. The enrichment broth method has been recommended to increase the sensitivity of detection of MRSA carriage. ${ }^{19}$ In our study, two of the six CA-MRSA strains were isolated only by the enrichment broth, and the use of this method resulted in improved recovery rates of $14 \%$.

As expected, all the $S$. aureus isolates, including the six CA-MRSA, were susceptible to most of the antimicrobial agents tested. Although most of these strains were sensitive to oxacillin, their $\mathrm{MIC}_{50}$ and $\mathrm{MIC}_{90}$ were near the resistance breakpoint to oxacillin (i.e., MIC $\geq 4 \mu \mathrm{g}$ / $\mathrm{mL}$ ), suggesting less potency and antimicrobial activity of this drug. This finding may be important for developing therapies for staphylococcal diseases.

In respect to mupirocin, the heavy growth with no visible zone of inhibition around the mupirocin disk observed in six $S$. aureus isolates may indicate a high level of resistance (i.e., MIC $\geq 1,024 \mu \mathrm{g} / \mathrm{mL}$ ) to this drug. ${ }^{20}$ This fact is worrisome because mupirocin is widely used for prevention of $S$. aureus intranasal colonization. ${ }^{1}$

Our results showed that all strains resistant to oxacillin by phenotypic methods carried the mecA gene. This was not observed in the oxacillin-sensitive $S$. aureus strains.

In conclusion, the results of this study showed that S. aureus nasal colonization is common in our student community, and suggest that CA-MRSA may be spreading in Brazil.

\section{ACKNOWLEDGEMENTS}

We thank the volunteers for their cooperation in the study, and Dr. Janet W. Reid for revising the English text.

This work was supported in part by the Coordenação de Aperfeiçoamento do Pessoal de Ensino Superior (CAPESBrazil). 


\section{REFERENCES}

1. Henderson DK. Managing methicillin-resistant staphylococci: a paradigm for preventing nosocomial transmission of resistant organisms. J Am Med 2006; 119: S45-52.

2. Naimi TS, LeDell KH, Como-Sabetti K et al. Comparison of community- and health care-associated methicillin-resistant Staphylococcus aureus infection. JAMA 2003; 290:2976-84.

3. Millar BC, Loughrev A, Elborn JS, Moore JE. Proposed definitions of community-associated meticillin-resistant Staphylococcus aureus (CA-MRSA). J Hosp Infect 2007; 67:109-13.

4. Fridkin SK, Hageman JC, Morrison M et al. Methicillin-resistant Staphylococcus aureus disease in three communities. N Engl J Med 2005; 352:1436-44.

5. Kluytmans J, van Belkum A, Verbrugh H. Nasal carriage of Staphylococcus aureus: epidemiology, underlying mechanisms, and associated risks. Clin Microbiol Rev 1997; 10:505-20.

6. Wertheim HF, Melles DC, Vos MC et al. The role of nasal carriage in Staphylococcus aureus infections. Lancet Infect Dis 2005; 5:751-62.

7. Warren DK, Guth RM, Coopersmith CM et al. Epidemiology of methicillin-resistant Staphylococcus aureus colonization in a surgical intensive care unit. Infect Control Hosp Epidemiol 2006; 27:1032-40.

8. Muñoz P, Hortal J, Gianella M et al. Nasal carriage of S. aureus increases the risk of surgical site infection after major heart surgery. J Hosp Infect 2007; 68:25-31.

9. Kuehnert MJ, Kruszon-Moran D, Hill HA et al. Prevalence of Staphylococcus aureus nasal colonization in the United States, 2001-2002. J Infect Dis 2006; 193:172-9.

10. Clinical and Laboratory Standards Institute. Performance standards for antimicrobial disk susceptibility test. Approved standard M2-A9 2006; Wayne, PA.

11. Clinical and Laboratory Standards Institute. Performance standards for antimicrobial susceptibility testing. Approved standard-S17 2007; Wayne, PA.
12. Finlay JE, Miller LA, Poupard JA. Interpretive criteria for testing susceptibility of staphylococci to mupirocin. Agents Chemother 1997; 41:1137-9.

13. Clinical and Laboratory Standards Institute. Methods for dilution antimicrobial susceptibility tests for bacteria that grow aerobically. Approved standard M7-A7 2006; Wayne, PA.

14. Munckhof WJ, Nimmo GR, Schooneveldt JM et al. Nasal carriage of Staphylococcus aureus, including communityassociated methicillin-resistant strains, in Queensland adults. Clin Microbiol Infect 2009; 15:149-55.

15. Stubbs E, Pegler M, Vickery A, Harbour C. Nasal carriage of Staphylococcus aureus in Australian (pre-clinical and clinical) medical students. J Hosp Infect 1994; 27:127-34.

16. Bischoff WE, Wallis ML, Tucker KB, Reboussin BA, Sherertz RJ. Staphylococcus aureus nasal carriage in a student community: prevalence, clonal relationships, and risk factors. Infect Control Hosp Epidemiol 2004; 25:485-91.

17. Ribeiro A, Dias C, Silva-Carvalho MC et al. First report of infection with community-acquired methicillin-resistant Staphylococcus aureus in South America. J Clin Microbiol 2005; 43:1985-8.

18. Kenner J, O'Connor T, Piantanida N et al. Rates of carriage of methicillin-resistant and methicillin-susceptible Staphylococcus aureus in an outpatient population. Infect Control Hosp Epidemiol 2003; 24:439-44.

19. Cookson B, Peters B, Webster M et al. Staff carriage of epidemic methicillin-resistant Staphylococcus aureus. J Clin Microbiol 1989; 27:1471-6.

20. Deshpande LM, Fix AM, Pfaller MA, Jones RN. Emerging elevated mupirocin resistance rates among staphylococcal isolates in the SENTRY Antimicrobial Surveillance Program (2000): correlations of results from disk diffusion, Etest and reference dilution methods. Diagn Microbiol Infect Dis 2002; 42:283-90. 